\title{
TM6SF2 E167K Variant Overexpression Promotes Expression of Inflammatory Cytokines in the HCC Cell Line HEPA 1-6
}

\author{
Shuixian Dü1, Songling Liao ${ }^{\# 2}$, Shousheng $\operatorname{Liu}^{3,5}$ and Yongning Xin*1,2,4,5 \\ ${ }^{1}$ Department of Infectious Disease, Qingdao Municipal Hospital, Qingdao, China; ${ }^{2}$ Department of Gastroenterology, Dalian \\ Medical University, Dalian, China; ${ }^{3}$ Central Laboratories, Qingdao Municipal Hospital, Qingdao, China; ${ }^{4}$ Department of \\ Gastroenterology, Qingdao Municipal Hospital, Qingdao, China; ${ }^{5}$ Digestive Disease Key Laboratory of Qingdao, Qingdao, China
}

\begin{abstract}
Background and Aims: Accumulated evidence has shown that chronic liver inflammation is one of the main risks of hepatocellular carcinoma ( $\mathrm{HCC})$, and E167K variant of the transmembrane 6 superfamily member 2 (TM6SF2) plays an important role in the progression of chronic liver diseases and HCC. The aim of this study was to explore effects of the TM6SF2 E167K variant on expression of the inflammatory cytokines TNF- $\alpha$, IL-2, IL- 6 and IL- 8 in the HCC cell line HEPA 1-6. Methods: HEPA 1-6 cells were infected with lentivirus containing either the TM6SF2 E167K variant or TM6SF2 wildtype, or control plasmids. Quantitative real-time polymerase chain reaction (qRT-PCR) and western blotting were conducted to analyze the expression of the inflammatory cytokines TNF- $\alpha$, IL-2, IL- 6 and IL-8. A $t$-test was used for statistical analysis. Results: Compared with the control group and TM6SF2 overexpression group, the relative expression of IL- 2 and IL- 6 mRNAs were significantly elevated in the TM6SF2 E167K overexpression group $(p<0.05)$. The relative mRNA expression of IL-8 in the TM6SF2 and TM6SF2 E167K overexpression groups were increased compared to the control group $(p<0.05)$. No obvious differences were observed for the expression of TNF- $\alpha$ in each group. The expression of TNF- $\alpha$, IL-2, IL- 6 and IL- 8 that was tested by western blotting showed the same trends as the qRT-PCR results. Conclusions: In conclusion, the E167K variant of the TM6SF2 gene could promote the expression of inflammatory cytokines IL-2 and IL- 6 in HEPA $1-6$ cells, suggesting that the TM6SF2 E167K variant may accelerate the progression of HCC.
\end{abstract}

Citation of this article: Du S, Liao S, Liu S, Xin Y. TM6SF2 E167K variant overexpression promotes expression of inflammatory cytokines in the HCC cell line HEPA 1-6. J Clin Transl Hepatol 2019;7(1):27-31. doi: 10.14218/JCTH.2018.00055.

Keywords: Hepatocellular carcinoma; TM6SF2; E167K variant; Inflammatory. Abbreviations: DMEM, Dulbecco's modified Eagle's medium; FBS, fetal bovine serum; GFP, green fluorescent protein; HCC, hepatocellular carcinoma; qRT-PCR, quantitative real-time polymerase chain reaction; SNP, single nucleotide polymorphism; TM6SF2, transmembrane 6 superfamily member 2 .

Received: 14 October 2018; Revised: 20 December 2018; Accepted: 27 December 2018

\#These two authors contributed equally to this work.

* Correspondence to: Yongning Xin, Department of Infectious Disease, Qingdao Municipal Hospital, 1 Jiaozhou Road, Qingdao, Shandong 266011, China. Tel: +86-532-82789463, Fax: +86-532-85968434, E-mail: xinyongning@163.com

\section{Introduction}

Hepatocellular carcinoma (HCC) is one of the most common malignancies with a high rate of metastasis-the main cause of death of the afflicted individuals-and has rates of morbidity and mortality that rank sixth and third in the world, respectively. ${ }^{1-3}$ In China, approximately 140,000 people die from HCC each year, accounting for about $50 \%$ of the HCC deaths worldwide. ${ }^{4}$ Chronic liver inflammation triggered by exposure to infectious agents (mainly by hepatitis $B$ virus) is regarded as the main risk factor of HCC progression (encompassing carcinogenesis, tumor growth and progression) that may relevant to the continuous, non-specific and inefficient activation of the immune system. ${ }^{5-7}$ The HCC inflammatory microenvironment consists of the immune cells and inflammatory cytokines; some cytokines, such as TNF- $\alpha$, IL-2, IL-6, and IL-8, are overexpressed in liver cancer and associated with risk of HCC and poor prognosis. ${ }^{8-11}$

A single nucleotide polymorphism (SNP) is a result of transition or transversion mutation of a single base, and is significantly associated with various genetic diseases. ${ }^{12}$ The transmembrane 6 superfamily member 2 (TM6SF2) is a rising star among molecules involved in lipid metabolism and chronic liver diseases, with increasing studies focusing on the important role of the TM6SF2 E167K variant in chronic liver disease and HCC. ${ }^{13-18}$ Yet, the relationship of the TM6SF2 E167K variant and the inflammatory response in liver remains unclear. To gain insight on this issue, we investigated the effect of the TM6SF2 E167K variant on expression of several cytokines (TNF- $\alpha$, IL-2, IL- 6 , and IL-8) in the HCC cell line HEPA 1-6.

In our study, HEPA 1-6 cells were infected with lentivirus containing either the TM6SF2 E167K variant or TM6SF2 wildtype, or control plasmids. The expression of mRNAs and proteins (TNF- $\alpha$, IL-2, IL- 6 , and IL-8) were tested in the TM6SF2 or TM6SF2 E167K overexpressing HEPA 1-6 cells.

The aim of this study was to explore effects of the TM6SF2 E167K variant on the expression of the inflammatory cytokines TNF- $\alpha$, IL-2, IL- 6 and IL- 8 in the HCC cell line HEPA 1-6.

\section{Methods}

\section{Cell culture}

HEPA 1-6 cells and 293T cells were cultured in Dulbecco's modified Eagle's medium (commonly known as DMEM) containing $100 \mu \mathrm{g} / \mathrm{mL}$ streptomycin (Gibcoß, USA), $100 \mathrm{U} / \mathrm{mL}$ penicillin and $10 \%$ fetal bovine serum (commonly known as 
Du S. et al: TM6SF2 E167K promotes inflammatory cytokines

Table 1. Sequences of the primers used in this study

\begin{tabular}{lll}
\hline Primer & Sequence, 5'-3' $^{\prime}$ & $\begin{array}{l}\text { Amplicon } \\
\text { size, bp }\end{array}$ \\
\hline P1 & ACCATTCAGGGGTCGTCAC & 131 \\
P2 & GGATGGTTCAGGGAGGTGTG & \\
P3 & TGCTGATGAGACAGCAACCA & 117 \\
P4 & GGCCTGATACGTTTAAGTGGG & \\
P5 & CTTCGGTCCAGTTGCCTTCT & 168 \\
P6 & TGGAATCTTCTCCTGGGGGT & \\
P7 & TGCTTCCCCTTAGCATTTGT & 101 \\
P8 & TGTGGAGGACACTACTGTTTT & \\
P9 & CTCCATCCTGGCCTCGCTGT & 202 \\
P10 & GCTGTCACCTTCACCGTTCC & \\
\hline
\end{tabular}

FBS; Hyclone, USA) in $25 \mathrm{~cm}^{2}$ plastic cell culture flasks (Corning, USA) at $37^{\circ} \mathrm{C}$ with $5 \% \mathrm{CO}_{2}$. When the cells reached approximately $80 \%$ confluence, the culture medium was removed, $1 \mathrm{~mL}$ trypsin was added to suspend the cells from the bottom of the flask for $1 \mathrm{~m}$ before $10 \mathrm{~mL}$ of new cell culture medium was added. The cells in suspension were divided into two new flasks equally and cultured for the next generation.

\section{Construction of lentiviral vectors}

Three lentiviral expression plasmids were constructed in this study: the blank control vector (pGC-FU-3FLAG-SV40Cherry), the TM6SF2 wild-type vector, and the TM6SF2 E167K variant vector. After $24 \mathrm{~h}$ of growth, the cultures in DMEM were supplemented with $10 \%$ FBS, after which the 293 T cells were transfected with the complexes, with the solution including the lentiviral vectors (expression plasmids and packaging plasmid) and Lipofectamine 2000 transfection reagent, and allowed to react for $48-72 \mathrm{~h}$. The culture medium was collected and concentrated, to serve as the lentiviral solution. The HEPA 1-6 cells were seeded into 6 -well plates $\left(2 \times 10^{5}\right.$ cells/well). After $24 \mathrm{~h}$ incubation, the cells were infected with lentiviral solution for $12 \mathrm{~h}$. The successfully transfected cells
Table 2. Primer amplification efficiencies

\begin{tabular}{llll}
\hline Primer & Slope & $R^{2}$ & E, \% \\
\hline P1/P2 & -3.427 & 0.998 & 95.43 \\
P3/P4 & -3.369 & 0.999 & 97.70 \\
P5/P6 & -3.381 & 0.998 & 97.24 \\
P7/P8 & -3.366 & 0.996 & 98.15 \\
P9/P10 & -3.405 & 0.998 & 96.34 \\
\hline
\end{tabular}

Primer amplification efficiencies (E) were calculated from the slope values of the standard curves according to the equation: $E=10^{(1 / \text {-slope })}-1$.

were validated by counting the percentage detected with green fluorescent protein (commonly known as GFP).

\section{qRT-PCR}

The effects of TM6SF2 or TM6SF2 E167K on the expression of inflammatory cytokines (TNF- $\alpha$, IL-2, IL- 6 , and IL-8) were analyzed by qRT-PCR. HEPA $1-6$ cells were collected after infection with the lentiviral solution for $48 \mathrm{~h}$, providing samples for overexpressed TM6SF2, TM6SF2 E167K expression, or negative control. Total RNA was harvested from the cells by using Trizol reagent (Invitrogen, USA). cDNAs were synthesized with the RevertAid First Strand cDNA Synthesis Kit (ThermoFisher, USA). The reaction was carried out at $42^{\circ} \mathrm{C}$ for $50 \mathrm{~m}$ and inactivated at $75^{\circ} \mathrm{C}$ for $15 \mathrm{~m}$. The cDNAs synthesized were stored at $-20^{\circ} \mathrm{C}$ until use. The primers $\mathrm{P} 1$ and P2 specific for TNF- $\alpha$, P3 and P4 specific for IL-2, P5 and P6 specific for IL-6, P7 and P8 specific for IL-8, and P9 and P10 specific for $\beta$-actin were designed using Primer Premier 5.0 program (Table 1 ).

The $\beta$-actin gene was chosen as the reference for internal standardization. The amplification efficiency of each primer pair was measured using the HEPA 1-6 cells' cDNA serially diluted 2-fold (Table 2 ). The qRT-PCR amplification was performed with a predenaturation step at $94^{\circ} \mathrm{C}$ for $10 \mathrm{~s}$, followed by 35 cycles of denaturation at $95^{\circ} \mathrm{C}$ for $10 \mathrm{~s}$, annealing at $58^{\circ} \mathrm{C}$ for $15 \mathrm{~s}$, extending at $72^{\circ} \mathrm{C}$ for $35 \mathrm{~s}$. The expression levels of TNF- $\alpha, \mathrm{IL}-2, \mathrm{IL}-6$, and IL- 8 relative to $\beta$-actin were calculated by the comparative CT method $\left(2^{-\Delta \Delta C T}\right){ }^{9}$
A

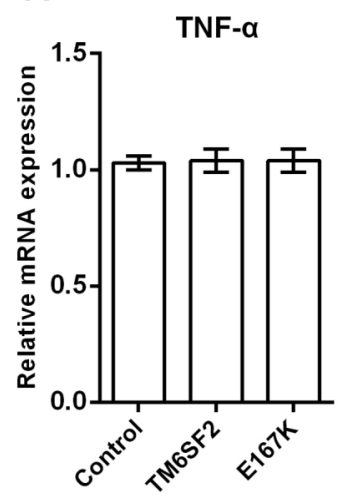

B

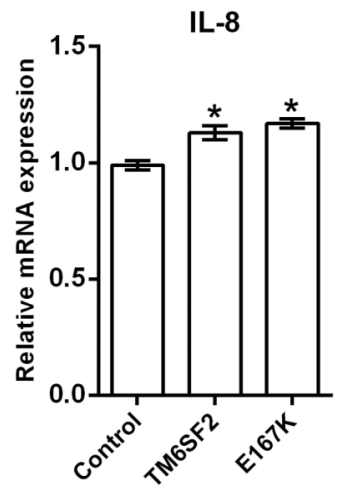

C

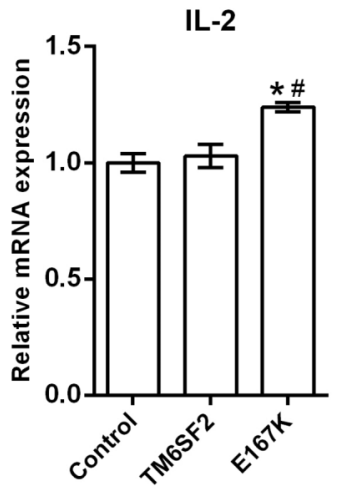

D

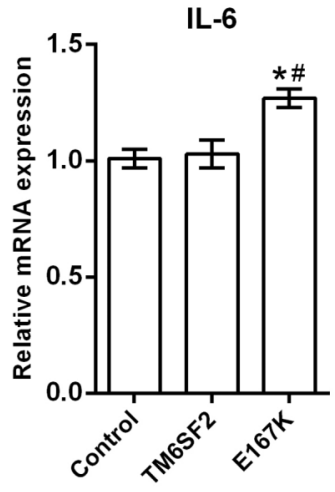

Fig. 1. Relative expression levels of TNF- $\alpha$, IL-2, IL-6 and IL-8 in HEPA 1-6 cells for the TM6SF2 overexpressed group, TM6SF2 E167K overexpressed group, and negative control group. Data are expressed as mean $\pm \mathrm{SD}$ from three replicates. The symbol $*$ indicates a statistical difference ( $p<0.05)$ compared to the control group. The symbol \# indicates a statistical difference $(p<0.05)$ compared to the TM6SF2 overexpressed group. 
Du S. et al: TM6SF2 E167K promotes inflammatory cytokines

A
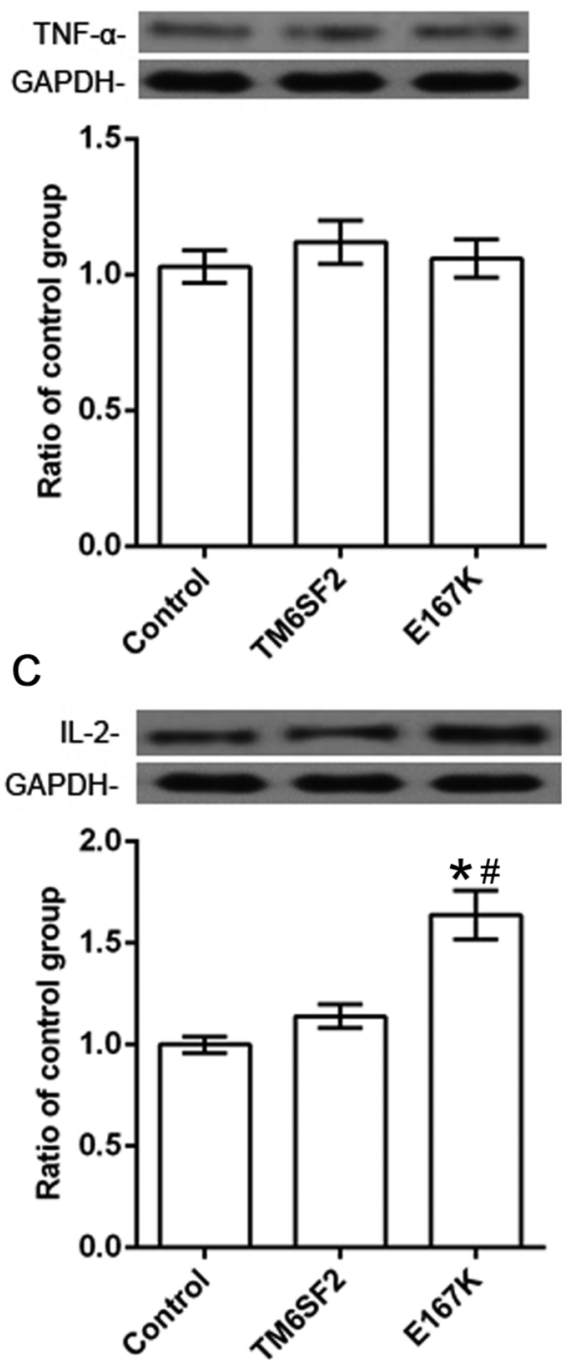

B
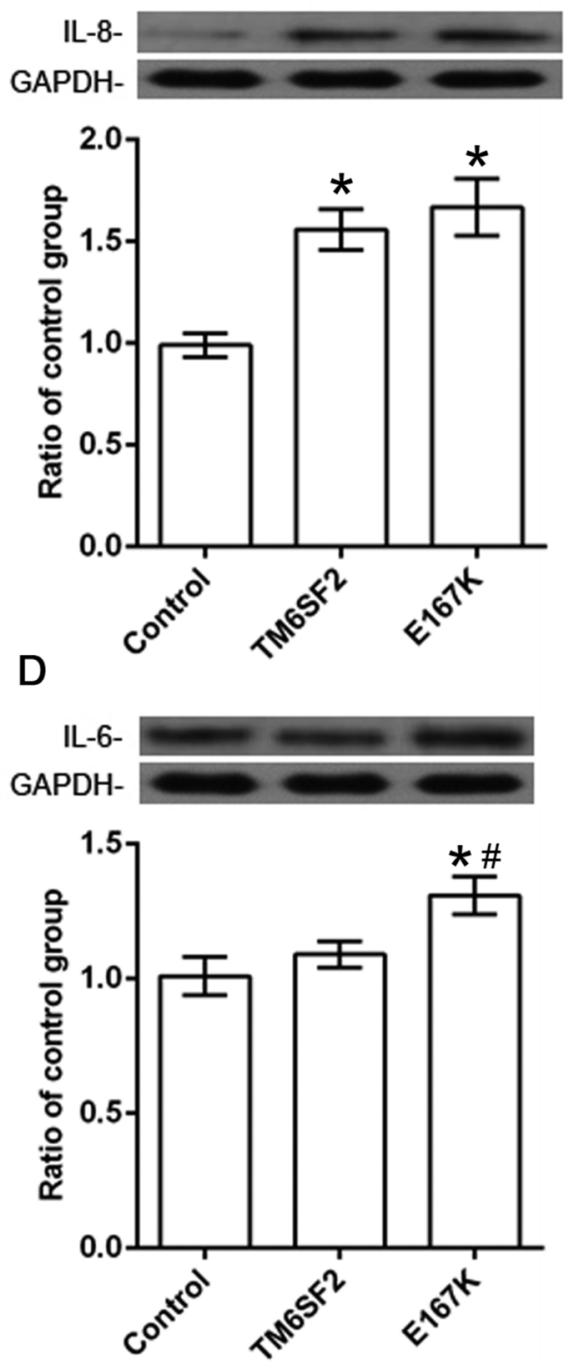

Fig. 2. Effects of TM6SF2 or TM6SF2 E167K on the expression levels of TNF- $\alpha$, IL-2, IL-6 and IL-8 in HEPA 1-6 cells. Bands were scanned and quantified using image analysis software, and results were corrected for protein loading by normalization for GAPDH expression. Data are presented as mean \pm SD from three replicates. The symbol * indicates a statistical difference $(p<0.05)$ compared to the control group. The symbol \# indicates a statistical difference $(p<0.05)$ compared to the TM6SF2 overexpressed group.

\section{Western blotting}

Western blotting was used to test the effects of TM6SF2 or TM6SF2 E167K on the protein expression of TNF- $\alpha$, IL-2, IL-6, and IL-8. HEPA 1-6 cells were collected after infection with the lentiviral solution for $48 \mathrm{~h}$, providing samples for overexpressed TM6SF2, TM6SF2 E167K expression, or negative control. RIPA buffer (Sigma-Aldrich, USA) was used to extract total protein from the HEPA 1-6 cells. Protein concentrations were determined by the Bradford method. Antibodies against TNF- $\alpha$ (KG22761; Jiangsu Keygen Biotech Co., Ltd., China), IL-2 (KG22463; Jiangsu Keygen Biotech Co., Ltd.), IL-6 (KG22468; Jiangsu Keygen Biotech Co., Ltd.), IL-8 (KG22469; Jiangsu Keygen Biotech Co., Ltd.) and GAPDH (KGAA002; Jiangsu Keygen Biotech Co., Ltd.) were used. The expression levels of TNF- $\alpha$, IL-2, IL- 6 and IL-8 proteins were normalized relative to the corresponding GAPDH (endogenous reference) level in each lane. The western blots were analyzed using GelPro Analyzer Version 4.5 software (Media Cybernetics, USA).

\section{Statistical analysis}

All the experiments were conducted at least three times. Data were expressed as the mean \pm standard deviation, and $t$-test was used for the statistical analysis. All the data were analyzed with SPSS 17.0 statistical software (SPSS Inc., USA). A $p$-value $<0.05$ was regarded as statistically significant in this study.

Results

\section{Overexpression of lentiviral vectors in HEPA 1-6 cells}

After the HEPA 1-6 cells had been infected with the lentiviral vectors for $48 \mathrm{~h}$, the positive rate of GFP was over $95 \%$ for all, 
suggesting that TM6SF2 and TM6SF2 E167K were overexpressed in HEPA 1-6 cells successfully.

\section{Expression profiles of TNF- $\alpha$, IL-2, IL-6 and IL-8 after overexpression of TM6SF2 or TM6SF2 E167K}

The relative expression profiles of TNF- $\alpha$, IL- 2, IL- 6 and IL- 8 in each group were determined by qRT-PCR. As the results showed, there were no significant differences in the expression of TNF- $\alpha$ in the TM6SF2 group or TM6SF2 E167K group compared to control $(p>0.05)$ (Fig. 1A). In the TM6SF2 and TM6SF2 E167K overexpressed groups, the expression of IL-8 was markedly increased compared to the control (both $p<$ 0.05 ), but no significant difference was observed between the TM6SF2 and TM6SF2 E167K overexpressed groups (Fig. 1B). There were no significant differences of IL- 2 and IL- 6 expression between the TM6SF2 overexpressed group and control, but in the TM6SF2 E167K overexpressed group, the expression levels of IL- 2 and IL- 6 were higher than in both the TM6SF2 overexpressed group and control (Fig. 1C and 1D).

\section{Detection of TNF- $\alpha, I L-2, I L-6$ and IL-8 protein expression by western blot}

Western blot was conducted to investigate the protein expression of TNF- $\alpha$, IL- 2 , IL- 6 and IL- 8 in the TM6SF2 and TM6SF2 overexpressed groups (Fig. 2A). No obvious differences of TNF- $\alpha$ protein expression were observed among the three groups (all $p>0.05$ ). In the TM6SF2 and TM6SF2 E167K overexpressed groups, the protein expression of IL-8 was higher than in the control $(p<0.05)$, but there was no significant difference between the TM6SF2 and TM6SF2 E167K overexpressed groups (Fig. 2B). The protein expression of both IL-2 and IL- 6 was markedly increased in the TM6SF2 E167K overexpressed group compared to the TM6SF2 overexpressed group and the control group (both $p<0.05$ ), but the expression was not higher between the TM6SF2 overexpressed group and the control group (Fig. 2C and 2D).

\section{Discussion}

Inflammatory cytokines play an important role in regulating the localization of inflammatory cells during the body's immune response. Inflammatory cytokines, which are secreted by tumor cells, could induce the migration of epithelial cells and immune cells in the circulation and immune response, facilitating involvement in the processes of angiogenesis, tumor growth, and metastasis. In addition, the recruitment of immune cells can also produce inflammatory cytokines that will regulate the progression of a tumor. ${ }^{19}$ The role of inflammatory cytokines has been confirmed in a variety of tumors, including those of breast cancer and cervical cancer among other tumor cells which are able to secrete CCL2 and CCL5 to promote the change of mononuclear cells to macrophages in a specific tumor site. ${ }^{20,21}$ These tumor-associated macrophages then secrete a variety of cytokines to regulate the formation of the local microenvironment, and participate in the processes of tumor cell growth, invasion, and metastasis. ${ }^{22}$

Effects of inflammatory cytokines on the progression of $\mathrm{HCC}$ has been studied widely. Chew et al. ${ }^{23}$ reported that TNF- $\alpha$ expression was related to the infiltration of Thl cells, CD8 (+) T cells and natural killer cells in HCC. Furthermore, they showed that TNF- $\alpha$ could promote the death of tumor cells and was associated with the progression and prognosis of HCC. Shin et al. ${ }^{24}$ found that IL-6, IL-1, and IL-12 were also prevalent inflammatory cytokines in the inflammatory microenvironment of HCC, and identified increased serum IL-6 level as a risk factor of HCC and as associated with the prognosis of HCC. The association between an elevated serum level of IL- 8 and the development of HCC has also been demonstrated by several studies. ${ }^{24-26}$ In addition, IL-2 has also been shown as a common inflammation cytokine in the inflammatory microenvironment of HCC. ${ }^{27-29}$

In this study, no significant difference was found for the TNF- $\alpha$ expression in HEPA 1-6 cells in response to overexpression of TM6SF2 or TM6SF2 E167K, suggesting that TM6SF2 may not regulate the TNF- $\alpha$ expression directly in HEPA 1-6 cells. However, we observed that the expression of both IL-2 and IL- 6 was significantly higher in the TM6SF2 E167K overexpressed group than in the TM6SF2 overexpressed or control groups of HEPA 1-6 cells. Finally, no significant difference was found for the expression of IL-8 in the TM6SF2 E167K and TM6SF2 overexpressed groups of HEPA 1-6 cells.

\section{Conclusions}

Collectively, we investigated the effect of TM6SF2 E167K on the expression levels of TNF- $\alpha$, IL-2, IL- 6 and IL- 8 in the HCC cell HEPA 1-6 in this study. Our data showed that overexpression of the TM6SF2 E167K protein significantly upregulates the expression of IL-2 and IL-6, although no significant up-regulations of TNF- $\alpha$ and IL- 8 were observed. Our findings suggest that the TM6SF2 E167K variant could promote the inflammation response and aggravate cell injury in HCC. Further studies are needed to illustrate the underlying mechanism and signaling pathway of TM6SF2 in the development of inflammation in HCC.

\section{Acknowledgments}

This study was supported by grants from the National Natural Science Foundation of China (No. 31770837), the Key Research Project of Shandong Province (No. 2016GSF201217), and the Qingdao, Shinan District Science and Technology Development Project Fund (No. 2016-3-016-YY).

\section{Conflict of interest}

The authors have no conflict of interests related to this publication.

\section{Author contributions}

Contributed to study concept and design (SD and $\mathrm{YX}$ ), acquisition of the data (SD and SoL), analysis and interpretation of the data (SD, SOL, and $\mathrm{ShL})$, drafting of the manuscript (SD and ShL), critical revision of the manuscript for important intellectual content ( $S h L$ and $Y X$ ), supervision ( $Y X)$. All the authors read and approved the final manuscript.

\section{References}

[1] Niu ZS, Niu XJ, Wang WH. Genetic alterations in hepatocellular carcinoma: An update. World J Gastroenterol 2016;22:9069-9095. doi: 10.3748/wjg. v22.i41.9069.

[2] Kakodkar R, Soin AS. Liver transplantation for HCC: A review. Indian J Surg 2012;74:100-117. doi: 10.1007/s12262-011-0387-2. 
[3] Hernandez-Gea V, Toffanin S, Friedman SL, Llovet JM. Role of the microenvironment in the pathogenesis and treatment of hepatocellular carcinoma. Gastroenterology 2013;144:512-527. doi: 10.1053/j.gastro.2013.01.002.

[4] Ji J, Wang H, Li Y, Zheng L, Yin Y, Zou Z, et al. Diagnostic evaluation of desgamma-carboxy prothrombin versus $\alpha$-fetoprotein for hepatitis $B$ virusrelated hepatocellular carcinoma in China: A large-scale, multicentre study. PLoS One 2016;11:e0153227. doi: 10.1371/journal.pone.0153227.

[5] Sarin SK, Kumar M, Lau GK, Abbas Z, Chan HL, Chen C], et al. Asian-Pacific clinical practice guidelines on the management of hepatitis B: a 2015 update. Hepatol Int 2016;10:1-98. doi: 10.1007/s12072-015-9675-4.

[6] Chang TS, Chen CL, Wu YC, Liu JJ, Kuo YC, Lee KF, et al. Inflammation promotes expression of stemness-related properties in HBV-related hepatocellular carcinoma. PLoS One 2016;11:e0149897. doi: 10.1371/journal.pone. 0149897.

[7] Jin K, Li T, Sánchez-Duffhues G, Zhou F, Zhang L. Involvement of inflammation and its related microRNAs in hepatocellular carcinoma. Oncotarget 2017;8:22145-22165. doi: 10.18632/oncotarget.13530.

[8] Jing Y, Han Z, Liu Y, Sun K, Zhang S, Jiang G, et al. Mesenchymal stem cells in inflammation microenvironment accelerates hepatocellular carcinoma metastasis by inducing epithelial-mesenchymal transition. PLoS One 2012 ; 7:e43272. doi: 10.1371/journal.pone.0043272.

[9] Shin SP, Kim NK, Kim JH, Lee JH, Kim JO, Cho SH, et al. Association between hepatocellular carcinoma and tumor necrosis factor alpha polymorphisms in South Korea. World J Gastroenterol 2015;21:13064-13072. doi: 10. 3748/wjg.v21.i46.13064.

[10] Liu F, Zhang W, Yang F, Feng T, Zhou M, Yu Y, et al. Interleukin-6-stimulated progranulin expression contributes to the malignancy of hepatocellular carcinoma cells by activating mTOR signaling. Sci Rep 2016;6:21260. doi: 10. 1038/srep21260.

[11] Wang Y, Wang W, Wang L, Wang X, Xia J. Regulatory mechanisms of interleukin- 8 production induced by tumour necrosis factor- $\alpha$ in human hepatocellular carcinoma cells. J Cell Mol Med 2012;16:496-506. doi: 10.1111/j. 1582-4934.2011.01337.x.

[12] Shaw V, Bullock K, Greenhalf W. Single-nucleotide polymorphism to associate cancer risk. Methods Mol Biol 2016;1381:93-110. doi: 10.1007/978-14939-3204-7 6.

[13] Kozlitina J, Smagris E, Stender S, Nordestgaard BG, Zhou HH, TybjærgHansen A, et al. Exome-wide association study identifies a TM6SF2 variant that confers susceptibility to nonalcoholic fatty liver disease. Nat Genet 2014;46:352-356. doi: 10.1038/ng.2901.

[14] Fan Y, Lu H, Guo Y, Zhu T, Garcia-Barrio MT, Jiang Z, et al. Hepatic transmembrane 6 superfamily member 2 regulates cholesterol metabolism in mice. Gastroenterology 2016;150:1208-1218. doi: 10.1053/j.gastro.2016.01.005.

[15] Eslam M, Mangia A, Berg T, Chan HL, Irving WL, Dore GJ, et al. Diverse impacts of the rs58542926 E167K variant in TM6SF2 on viral and metabolic liver disease phenotypes. Hepatology 2016;64:34-46. doi: 10.1002/hep. 28475.

[16] Stickel F, Buch S, Nischalke HD, Weiss KH, Gotthardt D, Fischer J, et al. Genetic variants in PNPLA3 and TM6SF2 predispose to the development of hepatocellular carcinoma in individuals with alcohol-related cirrhosis. Am J Gastroenterol 2018;113:1475-1483. doi: 10.1038/s41395-018-0041-8.

[17] Du S, Lu L, Miao Y, Jin W, Li C, Xin Y, et al. E167K polymorphism of TM6SF2 gene affects cell cycle of hepatocellular carcinoma cell HEPA 1-6. Lipids Health Dis 2017;16:76. doi: 10.1186/s12944-017-0468-8.

[18] Liu YL, Reeves HL, Burt AD, Tiniakos D, McPherson S, Leathart JB, et al. TM6SF2 rs58542926 influences hepatic fibrosis progression in patients with non-alcoholic fatty liver disease. Nat Commun 2014;5:4309. doi: 10. 1038/ncomms5309.

[19] Bishayee A. The role of inflammation and liver cancer. Adv Exp Med Biol 2014;816:401-435. doi: 10.1007/978-3-0348-0837-8_16.

[20] van der Sluis TC, Sluijter M, van Duikeren S, West BL, Melief C], Arens R, et al. Therapeutic peptide vaccine-induced CD8 $\mathrm{T}$ cells strongly modulate intratumoral macrophages required for tumor regression. Cancer Immunol Res 2015;3:1042-1051. doi: 10.1158/2326-6066.CIR-15-0052.

[21] Garcia CB, Fernandes PC, Micheli DC, Pereira AH, Murta EF, Tavares-Murta BM. Effect of treatment on mononuclear cell migration in cervical cancer patients. Tumori 2008;94:712-717. doi: 10.1177/030089160809400512.

[22] Castle PE, Phillips TM, Hildesheim A, Herrero R, Bratti MC, Rodríguez AC, et al. Immune profiling of plasma and cervical secretions using recycling immunoaffinity chromatography. Cancer Epidemiol Biomarkers Prev 2003; 12:1449-1456.

[23] Chew V, Chen J, Lee D, Loh E, Lee J, Lim KH, et al. Chemokine-driven lymphocyte infiltration: an early intratumoural event determining long-term survival in resectable hepatocellular carcinoma. Gut 2012;61:427-438. doi: 10 . 1136/gutjnl-2011-300509.

[24] Shin EC, Choi YH, Kim JS, Kim SJ, Park JH. Expression patterns of cytokines and chemokines genes in human hepatoma cells. Yonsei Med J 2002;43: 657-664. doi: 10.3349/ymj.2002.43.5.657.

[25] Hassanin OM, El-Masry TM, El-Baz A, Adel-Abou-Zaghla HM. Association of interleukin-8 gene polymorphisms and expression with hepatocellular carcinoma in Egyptian patients with liver cirrhosis. Egypt J Immunol 2017;24: 143-152.

[26] Liu Y, Zhang Y, Wang S, Dong QZ, Shen Z, Wang W, et al. Prospero-related homeobox 1 drives angiogenesis of hepatocellular carcinoma through selectively activating interleukin-8 expression. Hepatology 2017;66:1894-1909. doi: 10.1002/hep.29337.

[27] El-Shemi AG, Ashshi AM, Na Y, Li Y, Basalamah M, Al-Allaf FA, et al. Combined therapy with oncolytic adenoviruses encoding TRAIL and IL-12 genes markedly suppressed human hepatocellular carcinoma both in vitro and in an orthotopic transplanted mouse model. J Exp Clin Cancer Res 2016;35:74. doi: 10.1186/s13046-016-0353-8.

[28] Wei M, Marino J, Trowell A, Zhang H, Stromp Peraino J, Rajasekera PV, et al. Diphtheria toxin-based recombinant murine IL-2 fusion toxin for depleting murine regulatory T cells in vivo. Protein Eng Des Sel 2014;27:289-295. doi: 10.1093/protein/gzu034.

[29] Huang X, Qin J, Lu S. Kanglaite stimulates anticancer immune responses and inhibits HepG2 cell transplantation-induced tumor growth. Mol Med Rep 2014;10:2153-2159. doi: 10.3892/mmr.2014.2479. 